\title{
Exploiting group symmetry in truss topology optimization
}

\author{
Yanqin Bai • Etienne de Klerk • Dmitrii Pasechnik • \\ Renata Sotirov
}

Received: 23 February 2007 / Accepted: 29 May 2008 / Published online: 17 June 2008

(C) The Author(s) 2008. This article is published with open access at Springerlink.com

\begin{abstract}
We consider semidefinite programming (SDP) formulations of certain truss topology optimization problems, where a lower bound is imposed on the fundamental frequency of vibration of the truss structure. These SDP formulations were introduced in Ohsaki et al. (Comp. Meth. Appl. Mech. Eng. 180:203-217, 1999). We show how one may automatically obtain symmetric designs, by eliminating the 'redundant' symmetry in the SDP problem formulation. This has the advantage that the original SDP problem is substantially reduced in size for trusses with large symmetry groups.
\end{abstract}

Keywords Truss topology optimization · Semidefinite programming · Group symmetry

\section{Introduction}

In this paper we consider semidefinite programming (SDP) formulations of certain truss topology optimization problems. In particular, we consider so called groupsymmetric truss designs of the type studied by Kanno et al. (2001); see also Ohsaki et al. (1999), Ohsaki (2000).

\footnotetext{
Y. Bai

Shanghai University, Shanghai, China

E. de Klerk $(\varangle) \cdot$ R. Sotirov

Tilburg University, Tilburg, The Netherlands

e-mail: e.deklerk@uvt.nl

D. Pasechnik

Nanyang Technological University, Singapore, Singapore
} 
Kanno et al. pointed out that, although a symmetric truss design is desirable in practice, there may exist optimal solutions of the SDP formulation that do not exhibit this symmetry. They therefore proceed to show that certain search directions used in interior point algorithms for SDP preserve symmetry. This means that the interior point algorithms generate a sequence of iterates that are group symmetric, given that the starting point is group symmetric.

In this paper we show how one may automatically obtain symmetric designs, by eliminating the 'redundant' symmetry in the SDP problem formulation. In particular, we perform pre-processing to restrict the feasible set of the SDP problem to symmetric designs. This is in the spirit of work by Schrijver (1979), Gatermann and Parrilo (2004), de Klerk et al. (2006, 2007), and others, who have shown how 'group symmetric' SDP problems may be reduced in size using representation theory.

We illustrate our approach by considering a family of dome truss structures, and show that the resulting SDP problems may be greatly reduced in size via symmetry reduction.

\subsection{Outline}

We begin with a discussion of finite groups and their linear orthogonal representations in Sect. 2. The next two sections deal with matrix algebras and their representations. In particular, the case where the matrix algebra in question is the commutant of a linear representation of a finite group is of interest to us in this paper. In Sect. 5 we recall the notion of SDP problems with 'group symmetric data', and how these problems may be reduced in size using the algebraic techniques described in the previous sections. We then describe the SDP formulation of a truss topology optimization problem due to Ohsaki et al. (1999) in Sect. 6. We explain in which sense these SDP problems have group symmetric data, and show how they may be reduced using the techniques described in the previous section. Finally, we illustrate our results on a family of dome truss structures in Sect. 7.

\subsection{Notation}

The space of $p \times q$ real matrices is denoted by $\mathbb{R}^{p \times q}$, and the space of $k \times k$ symmetric matrices is denoted by $\mathcal{S}_{k}$, and the space of $k \times k$ positive semidefinite matrices by $\mathcal{S}_{k}^{+}$. We will sometimes also use the notation $X \succeq 0$ instead of $X \in \mathcal{S}_{k}^{+}$, if the order of the matrix is clear from the context.

We use $I_{n}$ to denote the identity matrix of order $n$, and omit the subscript if the order is clear from the context.

The Kronecker product $A \otimes B$ of matrices $A \in \mathbb{R}^{p \times q}$ and $B \in \mathbb{R}^{r \times s}$ is defined as the $p r \times q s$ matrix composed of $p q$ blocks of size $r \times s$, with block $i j$ given by $A_{i j} B$ $(i=1, \ldots, p),(j=1, \ldots, q)$.

The following properties of the Kronecker product will be used in the paper, see e.g. Graham (1981),

$$
\begin{gathered}
(A \otimes B)^{T}=A^{T} \otimes B^{T} \\
(A \otimes B)(C \otimes D)=A C \otimes B D,
\end{gathered}
$$


for all $A \in \mathbb{R}^{p \times q}, B \in \mathbb{R}^{r \times s}, C \in \mathbb{R}^{q \times k}, D \in \mathbb{R}^{s \times l}$.

Finally, let $E_{i j} \in \mathbb{R}^{n \times n}$ denote the matrix with 1 in position $i j$ and zero elsewhere.

\section{On finite groups and their representations}

The next definition recalls the fact that finite groups may be represented by multiplicative groups of orthogonal matrices.

Definition 1 (Miller 1972) Let $V$ be a real, $m$-dimensional vector space and identify $\mathbb{R}^{m \times m}$ (respectively, $\mathcal{O}_{m}$ ) as the space of all (respectively, orthogonal) $m \times m$ matrices. An (orthogonal) linear representation of a group $\mathcal{G}$ on $V$ is a group homomorphism $\mathcal{T}: \mathcal{G} \rightarrow \mathbb{R}^{m \times m}$ (respectively, $\mathcal{T}: \mathcal{G} \rightarrow \mathcal{O}_{m}$ ). In other words for each element $g \in \mathcal{G}$ there exists an invertible $\mathcal{T}_{g} \equiv \mathcal{T}(g) \in \mathbb{R}^{m \times m}$ (respectively, in $\mathcal{O}_{m}$ ) such that $\mathcal{T}\left(g_{1}\right) \mathcal{T}\left(g_{2}\right)=\mathcal{T}\left(g_{1} g_{2}\right)$.

In what follows we consider images of SDP data matrices $A_{i}=A_{i}^{T} \in \mathbb{R}^{m \times m}$ under $\mathcal{T}_{g}$ 's. Thus, we have to restrict our attention to orthogonal representations, as in the usual SDP setting one needs (as it will become clear in what follows) that $B_{i}=$ $\mathcal{T}_{g} A_{i} \mathcal{T}_{g}^{-1}$ are symmetric, i.e. $B_{i}=B_{i}^{T}$. From the representation-theoretic point of view, there is little loss of generality in considering such representations only. Indeed, any real linear representation of a finite group is equivalent, by conjugation with an upper-triangular matrix, to an orthogonal representation. ${ }^{1}$

The following theorem shows that, if one has two orthogonal representations of a finite group, one may obtain a third representation using Kronecker products. In representation theory this construction is known as tensor product of representations.

Theorem 2 Let $\mathcal{G}$ be a group and denote two orthogonal linear representations of $\mathcal{G}$ by $p_{i}(i=1, \ldots,|\mathcal{G}|)$ and $s_{i}(i=1, \ldots,|\mathcal{G}|)$, such that $p_{i}$ corresponds to $s_{i}(i=$ $1, \ldots,|\mathcal{G}|)$.

Then a third orthogonal linear representation of $\mathcal{G}$ is given by

$$
P_{i}:=p_{i} \otimes s_{i} \quad(i=1, \ldots,|\mathcal{G}|) .
$$

Proof Let indices $i, j, k \in\{1, \ldots,|\mathcal{G}|\}$ be given such that $p_{i} p_{j}=p_{k}$ (and therefore also $s_{i} s_{j}=s_{k}$ ). Note that

$$
\begin{aligned}
P_{i} P_{j} & =\left(p_{i} \otimes s_{i}\right)\left(p_{j} \otimes s_{j}\right) \\
& =\left(p_{i} p_{j}\right) \otimes\left(s_{i} s_{j}\right) \\
& =p_{k} \otimes s_{k} \equiv P_{k} .
\end{aligned}
$$

Moreover, note that the matrices $P_{i}$ are orthogonal, since the $p_{i}$ and $s_{i}$ 's are.

\footnotetext{
${ }^{1}$ This can be seen by modifying, in the obvious way, the standard representation theory argument, that works for any complex representation of $\mathcal{G}$, creating a $\mathcal{T}(\mathcal{G})$-invariant positive definite matrix $A=\sum_{g \in \mathcal{G}} \overline{\mathcal{T}(g)}^{T} \mathcal{T}(g)$, and conjugating $\mathcal{T}(\mathcal{G})$ by the Cholesky factors of $A$.
} 
The commutant (or centralizer ring) of $\mathcal{G}$ is defined by

$$
\mathcal{A}^{\prime}:=\left\{X \in \mathbb{R}^{n \times n}: X P=P X \quad \forall P \in \mathcal{G}\right\} .
$$

An alternative, equivalent, definition of the commutant is

$$
\mathcal{A}^{\prime}=\left\{X \in \mathbb{R}^{n \times n}: R(X)=X\right\},
$$

where

$$
R(X):=\frac{1}{|\mathcal{G}|} \sum_{P \in \mathcal{G}} P X P^{T}, \quad X \in \mathbb{R}^{n \times n}
$$

is called the Reynolds operator (or group average) of $\mathcal{G}$. Thus $R$ is the orthogonal projection onto the commutant. Orthonormal eigenvectors of $R$ corresponding to the eigenvalue 1 form a orthonormal basis of $\mathcal{A}^{\prime}$ (seen as a vector space).

The commutant is a $C^{*}$-algebra, i.e. a subspace of $\mathbb{R}^{n \times n}$ that is closed under matrix multiplication and conjugation.

We will study optimization problems where we may assume that the feasible set is contained in some commutant, and we therefore devote one more section to recall some results on representations of matrix $*$-algebras. The basic idea is that we want to obtain the most 'economical' representation of the feasible set of our optimization problem.

\section{Matrix algebras and their representations}

Let $\mathcal{A}_{1}$ and $\mathcal{A}_{2}$ denote two matrix $*$-algebras. We say that $\mathcal{A}_{1}$ and $\mathcal{A}_{2}$ are equivalent if there exists a unitary matrix $Q$ (i.e. $Q^{*} Q=I$ ) such that

$$
\mathcal{A}_{2}=\left\{Q^{*} X Q: X \in \mathcal{A}_{1}\right\} \text {. }
$$

We define the direct sum of matrices $X_{1}$ and $X_{2}$ as

$$
X_{1} \oplus X_{2}:=\left(\begin{array}{cc}
X_{1} & 0 \\
0 & X_{2}
\end{array}\right) .
$$

An algebra $\mathcal{A}$ is called basic if

$$
\mathcal{A}=\left\{\bigoplus_{i=1}^{t} M: M \in \mathbb{C}^{m \times m}\right\}
$$

for some $t$ and $m$. Finally, the direct sum of two algebras $\mathcal{A}_{1}$ and $\mathcal{A}_{2}$ is defined as

$$
\mathcal{A}_{1} \oplus \mathcal{A}_{2}:=\left\{X_{1} \oplus X_{2}: X_{1} \in \mathcal{A}_{1}, X_{2} \in \mathcal{A}_{2}\right\}
$$

The following existence theorem gives the so-called completely reduced representation of a matrix $*$-algebra $\mathcal{A}$. 
Theorem 3 (Wedderburn 1907) ${ }^{2}$ Each matrix *-algebra is equivalent to a direct sum of basic algebras and a zero algebra.

In general this completely reduced representation is not known, but it is known in our case, that is, when our $*$-algebra is the commutant ${ }^{3}$ of a finite group representation.

In the next section we give details on how to compute the completely reduced representation of the commutant. The reader may wish to skip this section during a first reading of the paper.

\section{Commutant of a group representation}

Here we summarize (and use) the relevant material from Serre (1977), in particular from Sect. 13.2, where $\mathbb{R}$-representations are treated. ${ }^{4}$

Let $\mathbb{F}$ be either $\mathbb{C}$ or $\mathbb{R}$, and $\mathcal{T}$ be a $\mathbb{F}$-linear representation of a finite group $\mathcal{G}$ into $\mathbb{F}^{m \times m}$, or more precisely, into the group $G L_{m}(\mathbb{F})$ of the invertible matrices in $\mathbb{F}^{m \times m}$. The character of $\mathcal{T}$ is a function $\chi_{\mathcal{T}}: \mathcal{G} \rightarrow \mathbb{F}$ given by $\chi_{\mathcal{T}}(g):=\operatorname{tr}(\mathcal{T}(g))$, that encodes a lot of information about $\mathcal{T}$ and $\mathcal{G}$. For instance, two representations of $\mathcal{G}$ are equivalent if and only if they have the same character. Note that in order to know $\chi_{\mathcal{T}}$, it suffices to know its values on representatives of conjugacy classes $^{5}$ of $\mathcal{G}$.

Important (and easy to check) formulae for the characters of the direct sum $\mathcal{T} \oplus \mathcal{Q}$ and of the tensor product $\mathcal{T} \otimes \mathcal{Q}$ of two representations $\mathcal{T}$ and $\mathcal{Q}$ of $\mathcal{G}$ are as follows:

$$
\chi_{\mathcal{T} \oplus \mathcal{Q}}(g)=\chi_{\mathcal{T}}(g)+\chi_{\mathcal{Q}}(g), \quad \chi_{\mathcal{T} \otimes \mathcal{Q}}(g)=\chi_{\mathcal{T}}(g) \chi_{\mathcal{Q}}(g), \quad g \in \mathcal{G} .
$$

A representation is called irreducible if the space $\mathbb{F}^{m}$ does not contain a proper subspace that is left invariant by all the $\mathcal{T}(g)$, where $g \in \mathcal{G}$. The following is wellknown, cf. e.g. Serre (1977, p. 108).

Theorem 4 The commutant $C(\mathcal{I})$ of a linear $\mathbb{R}$-irreducible representation $\mathcal{I}$ of $\mathcal{G}$ is isomorphic to a division ring ${ }^{6}$ over $\mathbb{R}$. Thus $C(\mathcal{I})$ depends upon the decomposition of the representation $\mathcal{I}$ over $\mathbb{C}$. Namely:

1. I is irreducible over $\mathbb{C}: C(\mathcal{I}) \cong \mathbb{R}, \operatorname{dim}_{\mathbb{R}}(C(\mathcal{I}))=1$.

2. $\chi_{\mathcal{G}}(\mathcal{I})=\zeta_{\mathcal{G}}(\mathcal{J})+\overline{\zeta_{\mathcal{G}}(\mathcal{J})}$, with $\mathbb{C}$-valued $\zeta_{\mathcal{G}}(\mathcal{J})$ and a $\mathbb{C}$-representation $\mathcal{J}$ of $\mathcal{G}$ : $C(\mathcal{I}) \cong \mathbb{C}, \operatorname{dim}_{\mathbb{R}}(C(\mathcal{I}))=2$.

\footnotetext{
${ }^{2}$ A detailed treatment of this material is given in Wedderburn (1934); our statement of the theorem is based on lecture notes by A. Schrijver, available at http://homepages.cwi.nl/ lex/files/block.pdf.

${ }^{3}$ Called commuting algebra in Serre (1977).

${ }^{4}$ Representation theory of finite groups is easiest over the field of complex numbers $\mathbb{C}$. It is in fact beneficial to bear in mind that every $\mathbb{R}$-representation is also a $\mathbb{C}$-representation.

${ }^{5}$ The conjugacy class of $g \in \mathcal{G}$ in $\mathcal{G}$ is the set $\left\{h g h^{-1} \mid h \in \mathcal{G}\right\} \subset \mathcal{G}$.

${ }^{6} \mathrm{~A}$ division ring is an algebraic object that is "just like" a field, except that its multiplication need not be commutative. The finite-dimensional division rings that have $\mathbb{R}$ in the center are classified: such a ring is either $\mathbb{R}$, or $\mathbb{C}$, or $\mathbb{H}$. The latter is the famous Hamilton's algebra of quaternions.
} 
3. $\chi_{\mathcal{G}}(\mathcal{I})=2 \zeta_{\mathcal{G}}(\mathcal{J})$, with a $\mathbb{R}$-valued $\zeta_{\mathcal{G}}(\mathcal{J})$ and a $\mathbb{C}$-representation $\mathcal{J}$ of $\mathcal{G}: C(\mathcal{I}) \cong$ $\mathbb{H}, \operatorname{dim}_{\mathbb{R}}(C(\mathcal{I}))=4$.

In our case the group $\mathcal{G}$ will only have irreducible over $\mathbb{R}$ representations that remain irreducible over $\mathbb{C}$, so only case 1 will occur. One can describe $C(\mathcal{I})$ explicitly. In case 1 it just consists of the scalar matrices $\lambda I, \lambda \in \mathbb{R}$.

For the curious reader, let us give an example of case 2 . Let $\mathcal{G}=\mathbb{Z}_{n}$, the cyclic group of order $n$, and let $a$ be generator of the cyclic group as an abstract group.

All its irreducible representations over $\mathbb{C}$ are 1-dimensional. However $\mathbb{Z}_{n}=\left\{a^{k} \mid\right.$ $k=1, \ldots, n\}$ has 2 -dimensional representations over $\mathbb{R}$ that are irreducible over $\mathbb{R}$, e.g.

$$
a^{k} \mapsto\left(\begin{array}{cc}
\cos 2 \pi k / n & \sin 2 \pi k / n \\
-\sin 2 \pi k / n & \cos 2 \pi k / n
\end{array}\right) .
$$

This representation $\mathcal{I}$ has the character $\chi\left(a^{k}\right)=2 \cos 2 \pi k / n=e^{2 \pi \mathbf{i} k / n}+e^{-2 \pi \mathbf{i} k / n}=$ $\zeta\left(a^{k}\right)+\overline{\zeta\left(a^{k}\right)}$, where $\zeta$ is the character of the 1 -dimensional $\mathbb{C}$-representation $a^{k} \mapsto$ $e^{2 \pi \mathbf{i} k / n}$. We can directly check that

$$
C(\mathcal{I})=\left\langle\left(\begin{array}{ll}
y & 0 \\
0 & y
\end{array}\right),\left(\begin{array}{cc}
0 & z \\
-z & 0
\end{array}\right), y, z \in \mathbb{R}\right\rangle .
$$

Going back to the general situation, $\mathcal{T}$ is equivalent to a direct sum of irreducible $\mathbb{F}$-representations $\mathcal{T}_{k}$, i.e.

$$
\mathcal{T}(g) \cong \mathcal{T}_{1}(g) \oplus \mathcal{T}_{2}(g) \oplus \cdots \oplus \mathcal{T}_{\ell}(g), \quad g \in \mathcal{G}
$$

On the other hand, $\mathcal{G}$ has exactly as many irreducible $\mathbb{C}$-representations as it has conjugacy classes, say $c:=c_{\mathbb{C}}$. Theorem 4 implies that when $\mathbb{F}=\mathbb{R}$ then the number $c_{\mathbb{F}}$ of $\mathbb{R}$-irreducible representations is at most $c_{\mathbb{C}}$. By rearranging, if necessary, direct summands, and abbreviating

$$
k \mathcal{J}(g)=\underbrace{\mathcal{J}(g) \oplus \cdots \oplus \mathcal{J}(g)}_{k \text { times }},
$$

we obtain a decomposition that is called explicit in Serre (1977, Sect. 2.7).

$$
\mathcal{T}(g) \cong m_{1} \mathcal{T}_{1}(g) \oplus m_{2} \mathcal{T}_{2}(g) \oplus \cdots \oplus m_{c} \mathcal{T}_{c}(g), \quad m_{i} \geq 0,1 \leq i \leq c, g \in \mathcal{G},
$$

where $\mathcal{T}_{i}$ is not equivalent to $\mathcal{T}_{j}$ when $i \neq j$. The latter implies in particular that any $x \in C(\mathcal{T})$ must respect the coarse block structure provided by the $\mathcal{T}_{k}$,s; on the other hand $x$ can have a nontrivial action within any $m_{k} \mathcal{T}_{k}$-block. The following completely describes the commutant of such a block.

Theorem 5 Let $\mathcal{I}$ be an irreducible $\mathbb{R}$-representation of $\mathcal{G}$. Then for any $k \geq 1$ one has $C(k \mathcal{I})=M_{k}(\mathbb{R}) \otimes C(\mathcal{I})$, where $C(\mathcal{I})$ is isomorphic to either $\mathbb{R}, \mathbb{C}$, or $\mathbb{H}$, depending upon $\mathcal{I}$ in accordance with Theorem 4. 
The formula $C(k \mathcal{I})=M_{k}(\mathbb{R}) \otimes C(\mathcal{I})$ just says that each element $x \in C(k \mathcal{I})$ equals the Kronecker product $x=X \otimes Y$, with $X \in M_{k}(\mathbb{R})$ and $Y \in C(\mathcal{I})$. In the case 1 of Theorem 4 , we have $Y=\lambda I, \lambda \in \mathbb{R}$.

Further, we will use the following extremely useful First Orthogonality Relation for characters, see Serre (1977, Theorem I.3). Let $\chi, \zeta$ be characters of two representations of $\mathcal{G}$, and define the scalar product of them to be

$$
\langle\chi \mid \zeta\rangle=\frac{1}{|\mathcal{G}|} \sum_{g \in \mathcal{G}} \chi(g) \overline{\zeta(g)}
$$

Theorem 6 Let $\chi, \zeta$ be characters of two nonequivalent irreducible representations of $\mathcal{G}$. Then $\langle\chi \mid \zeta\rangle=0$, and $\langle\chi \mid \chi\rangle=1$.

\subsection{Computing the decomposition}

Computing the decomposition (4), more precisely, the isomorphism, that is, a matrix $M$ such that $M^{-1} \mathcal{T}(g) M$ has the form as in (4), between the original representation and the representation (4) is greatly helped by the explicit knowledge of each irreducible occurring there. Let $W:=\mathcal{T}_{k}$ for some $k$ be an irreducible representation of $\mathcal{G}$ of dimension $\ell$, given by the matrices $\left(w_{i j}(g)\right)$ for each $g \in \mathcal{G}$. For each $1 \leq \alpha, \beta \leq \ell$ consider the linear map

$$
p_{\alpha \beta}=\frac{\ell}{|\mathcal{G}|} \sum_{g \in \mathcal{G}} w_{\beta \alpha}\left(g^{-1}\right) \mathcal{T}(g),
$$

described in Serre (1977, Sect. 2.7), see in particular Prop. 8 there. In particular $p_{\alpha \alpha}$ is a projection. Denote its image by $V_{\alpha}$. Then $V_{\alpha} \cap V_{\beta}=\{0\}$ for $\alpha \neq \beta$. Moreover $\operatorname{dim} V_{\alpha}=m_{k}$ and $\operatorname{dim} V_{1} \oplus \cdots \oplus V_{\ell}=m_{k} \ell$. The matrices $\mathcal{T}(g), g \in \mathcal{G}$, preserve $V=V_{1} \oplus \cdots \oplus V_{\ell}$. Such subspaces are called $\mathcal{G}$-stable, because $\mathcal{G}$, or, more precisely $\mathcal{T}(g)$ for any $g \in \mathcal{G}$, maps each vector in $V$ to a vector in $V$. Each $\mathcal{T}(g)$ on $V$ is equivalent to $m_{k} W(g)$.

It remains to specify the $m_{k}$ subspaces $V^{s}$ of $V$ that are $\mathcal{G}$-stable, so that on each of them $\mathcal{T}(g)$ is equivalent to $W(g)$. Let $V_{1}$ be spanned by $x_{1}, \ldots, x_{m_{k}}$. Then for $1 \leq s \leq m_{k}$ the subspace is spanned by $p_{11}\left(x_{s}\right), p_{21}\left(x_{s}\right), \ldots, p_{\ell, 1}\left(x_{s}\right)$.

With this information at hand, it is a routine linear algebra to write down an isomorphism $M$. As we know the natural basis of the commutant for the representation in the form as at the right-hand side of (4), we can apply $M^{-1}$ to it to obtain a basis for the commutant of $\mathcal{T}$.

\section{Group symmetric SDP problems}

Assume that the following semidefinite programming problem is given

$$
p^{*}:=\min _{X \succeq 0}\left\{\operatorname{tr}\left(A_{0} X\right): \operatorname{tr}\left(A_{k} X\right)=b_{k}, k=1, \ldots, m\right\},
$$


where $A_{i} \in \mathcal{S}_{n}(i=0, \ldots, m)$ are given. The associated dual problem is

$$
p^{*}=\max _{y \in \mathbb{R}^{m}}\left\{b^{T} y: A_{0}-\sum_{i=1}^{m} y_{i} A_{i} \succeq 0\right\} .
$$

We assume that both problems satisfy the Slater condition so that both problems have optimal solutions with identical optimal values.

Assumption 1 (Group symmetry) We assume that there is a nontrivial multiplicative group of orthogonal matrices $\mathcal{G}$ such that the associated Reynolds operator

$$
R(X):=\frac{1}{|\mathcal{G}|} \sum_{P \in \mathcal{G}} P X P^{T}, \quad X \in \mathbb{R}^{n \times n}
$$

maps the feasible set of (5) into itself and leaves the objective value invariant, i.e.

$$
\operatorname{tr}\left(A_{0} R(X)\right)=\operatorname{tr}\left(A_{0} X\right) \text { if } X \text { is a feasible point of (5). }
$$

Since the Reynolds operator maps the convex feasible set into itself and preserves the objective values of feasible solutions, we may restrict the optimization to feasible points in the centralizer ring (commutant) $\mathcal{A}^{\prime}$ of $\mathcal{G}$.

Moreover, the next result shows that one may replace the data matrices $A_{i}(i=$ $0, \ldots, m)$ in the SDP formulation (5) by their projections $R\left(A_{i}\right)(i=0, \ldots, m)$ onto the centralizer ring.

\section{Theorem 7 One has}

$$
p^{*}=\min _{X \succeq 0}\left\{\operatorname{tr}\left(R\left(A_{0}\right) X\right): \operatorname{tr}\left(R\left(A_{k}\right) X\right)=b_{k}, k=1, \ldots, m\right\}
$$

Proof The proof is an immediate consequence of Assumption 1 and the observation that $\operatorname{tr}\left(A_{i} R(X)\right)=\operatorname{tr}\left(R\left(A_{i}\right) X\right)$ for any $i$.

It follows that one may also replace the data matrices in the dual problem (6) by their projections onto the centralizer ring.

Corollary 8 Under Assumption 1, one has

$$
p^{*}=\max _{y \in \mathbb{R}^{m}}\left\{b^{T} y: R\left(A_{0}\right)-\sum_{i=1}^{m} y_{i} R\left(A_{i}\right) \succeq 0\right\} .
$$

If the completely reduced representation of the centralizer ring is known, this may be used to reduce the size of the SDP problem, by block-diagonalizing the matrix variable $S:=R\left(A_{0}\right)-\sum_{i=1}^{m} y_{i} R\left(A_{i}\right)$ using the procedure described in the previous section. 
This idea has been applied most notably by Schrijver (1979, 2005), for SDP's arising from coding theory, where the centralizer ring is either the Bose-Mesner algebra (Schrijver 1979), or the Terwilliger algebra of the Hamming scheme (Schrijver 2005).

We will use the same approach below for an example in truss topology optimization.

\section{A truss topology optimization problem}

We consider a truss defined by a ground structure of nodes and bars. Let $m$ be the number of bars, and assume that free nodes have 3 degrees of freedom.

Let $b \in \mathbb{R}^{m}$ be the vector of bar lengths, and $z \in \mathbb{R}^{m}$ the vector of cross-sectional areas. The topology optimization problem (TOP) is to find a truss of minimum volume such that the fundamental frequency of vibration is higher than some prescribed critical value (Kanno et al. 2001):

$$
\begin{array}{ll}
\text { (TOP) } \min & \sum_{i=1}^{m} b_{i} z_{i} \\
\text { s.t. } & S=\sum_{i=1}^{m}\left(K_{i}-\bar{\Omega} M_{i}\right) z_{i}-\bar{\Omega} M_{0} \\
& z_{i} \geq 0, \quad i=1, \ldots, m \\
& S \succeq 0,
\end{array}
$$

where $\bar{\Omega}$ is a lower bound on the (squared) fundamental frequency of vibration of the truss, and $M_{0}$ the so-called non-structural mass matrix. If a nonstructural mass $m_{i}$ is added to each free node $i$, then the nonstructural mass matrix $M_{0}$ is given by

$$
M_{0}:=\frac{1}{3} \sum_{i \text { is a free node }} m_{i} E_{i i} \otimes I_{3} .
$$

The matrices $z_{i} K_{i}$ and $z_{i} M_{i}$ are known as element stiffness and element mass matrices, respectively. If a bar $k$ has endpoints $i$ and $j$, these matrices are defined as follows:

If $i$ and $j$ are free nodes, then

$$
M_{k}=\frac{\rho b_{k}}{6}\left(2\left(E_{i i}+E_{j j}\right)+E_{i j}+E_{j i}\right) \otimes I_{3},
$$

where $\rho$ is the mass density of the bars, and $b_{k}$ the length of bar $k$; moreover

$$
K_{k}=\frac{\kappa}{b_{k}^{2}}\left(E_{i i}+E_{j j}-E_{i j}-E_{j i}\right) \otimes d_{k} d_{k}^{T},
$$

where $d_{k}$ is a unit direction vector of the bar $k$, and $\kappa$ the elastic modulus (Young's modulus). 
If $i$ is a free and $j$ a fixed node, then

$$
M_{k}=\frac{\rho b_{k}}{6}\left(2 E_{i i}\right) \otimes I_{3},
$$

and

$$
K_{k}=\frac{\kappa}{b_{k}^{2}} E_{i i} \otimes d_{k} d_{k}^{T}
$$

Note that the order of the matrices $E_{i j}$ equals the number of free nodes.

\subsection{Truss symmetry}

We may formally define the symmetry group of a given truss, by viewing the ground structure of the truss as the embedding of a graph in $\mathbb{R}^{3}$ (for space trusses) or in $\mathbb{R}^{2}$ (for plane trusses). We will in fact only consider space trusses in this paper.

Now the symmetry group of the truss is defined as the subgroup of graph automorphisms that:

1. Are also isometries (i.e. that also preserve edge (i.e. bar) lengths);

2. Map free nodes to free nodes with the same nonstructural mass and fixed nodes to fixed nodes.

We will consider a subgroup, say $\mathcal{G}$, of the symmetry group of the truss, by only considering the free nodes. This is convenient, since the fixed nodes do not appear in the data of problem (TOP). It will be useful to represent $\mathcal{G}$ in two different ways:

1. As a multiplicative group of $3 \times 3$ orthogonal matrices $r_{i}(i=1, \ldots,|\mathcal{G}|)$ that are bijections of the set of coordinates of the nodes to itself; in other words, the $r_{i}$ matrices are rotation or reflection matrices.

2. As a group of permutation matrices $p_{i}(i=1, \ldots,|\mathcal{G}|)$ corresponding to the permutations of the free nodes in the automorphisms.

Lemma 9 The matrices $P_{i}:=p_{i} \otimes r_{i}(i=1, \ldots,|\mathcal{G}|)$ form an orthogonal, linear representation of $\mathcal{G}$.

Proof Follows immediately from Theorem 2.

Theorem 10 If $K_{j}$ (resp. $M_{j}$ ) corresponds to an element stiffness (resp. mass) matrix of the truss structure, and an index $i \in\{1, \ldots,|\mathcal{G}|\}$ is given, then there is an index $k$ such that

$$
P_{i} K_{j} P_{i}^{T}=K_{k}
$$

resp.

$$
P_{i} M_{j} P_{i}^{T}=M_{k}
$$

Moreover, one has $b_{j}=b_{k}$. Finally, one has

$$
P_{i} M_{0} P_{i}^{T}=M_{0}
$$

where $M_{0}$ is the nonstructural mass matrix of the truss as defined in (7). 
Proof If $i$ and $j$ are free nodes and the endpoints of bar $k$, then the matrix $M_{k}$ is given by (8).

Now let $p$ be a permutation matrix corresponding to a permutation of the nodes, and let $r$ be the corresponding $3 \times 3$ rotation/reflection matrix. Set $P=p \otimes r$.

One has

$$
\begin{aligned}
P M_{k} P^{T} & =\frac{\rho b_{k}}{6}(p \otimes r)\left(\left(2\left(E_{i i}+E_{j j}\right)+E_{i j}+E_{j i}\right) \otimes I_{3}\right)\left(p^{T} \otimes r^{T}\right) \\
& =\frac{\rho b_{k}}{6}(p \otimes r)\left(\left(2\left(E_{i i}+E_{j j}\right)+E_{i j}+E_{j i}\right) p^{T} \otimes r^{T}\right) \\
& =\frac{\rho b_{k}}{6}\left(p\left(2\left(E_{i i}+E_{j j}\right)+E_{i j}+E_{j i}\right) p^{T}\right) \otimes\left(r r^{T}\right) \\
& =\frac{\rho b_{k}}{6}\left(p\left(2\left(E_{i i}+E_{j j}\right)+E_{i j}+E_{j i}\right) p^{T}\right) \otimes I_{3} .
\end{aligned}
$$

Assume now that $p$ maps bar $k=(i, j)$ to the bar $k^{\prime}=\left(i^{\prime}, j^{\prime}\right)$. Since $b_{k}=b_{k^{\prime}}$ and

$$
p\left(2\left(E_{i i}+E_{j j}\right)+E_{i j}+E_{j i}\right) p^{T}=\left(2\left(E_{i^{\prime} i^{\prime}}+E_{j^{\prime} j^{\prime}}\right)+E_{i^{\prime} j^{\prime}}+E_{j^{\prime} i^{\prime}}\right),
$$

we have $P M_{k} P^{T}=M_{k^{\prime}}$.

If $i$ is a free and $j$ a fixed node, then $M_{k}$ is given by (10), and the proof is similar to the previous case, as is the proof of (12).

The proof for the element stiffness matrices is also similar: Let $z_{k} K_{k}$ be an element stiffness matrix corresponding to a bar $(i, j)$. If $i$ and $j$ are free nodes, then using (9) one has

$$
\begin{aligned}
P K_{k} P^{T} & =\frac{\kappa}{b_{k}^{2}}(p \otimes r)\left(\left(E_{i i}+E_{j j}-E_{i j}-E_{j i}\right) \otimes d_{k} d_{k}^{T}\right)\left(p^{T} \otimes r^{T}\right) \\
& =\frac{\kappa}{b_{k}^{2}}(p \otimes r)\left(\left(E_{i i}+E_{j j}-E_{i j}-E_{j i}\right) p^{T} \otimes d_{k} d_{k}^{T} r^{T}\right) \\
& =\frac{\kappa}{b_{k}^{2}}\left(p\left(E_{i i}+E_{j j}-E_{i j}-E_{j i}\right) p^{T}\right) \otimes\left(\left(r d_{k}\right)\left(r d_{k}\right)^{T}\right) .
\end{aligned}
$$

Now use the fact that $r d_{k}$ is the direction vector of bar $k^{\prime}:=\left(i^{\prime}, j^{\prime}\right)$, say $d_{k^{\prime}}$, i.e.

$$
P K_{k} P^{T}=\frac{\kappa}{b_{k^{\prime}}^{2}}\left(E_{i^{\prime} i^{\prime}}+E_{j^{\prime} j^{\prime}}-E_{i^{\prime} j^{\prime}}-E_{j^{\prime} i^{\prime}}\right) \otimes d_{k^{\prime}}\left(d_{k^{\prime}}\right)^{T}=: K_{k^{\prime}}
$$

If $i$ is a free node and $j$ a fixed node, then $K_{k}$ is given by (11), and the proof is similar as before.

Thus the dual problem of (TOP) satisfies Assumption 1 for the representation $P_{1}, \ldots, P_{|\mathcal{G}|}$ of the symmetry group $\mathcal{G}$. 
Corollary 11 Consider the orthogonal, linear representation of the truss symmetry group $\mathcal{G}$ given by $P_{i}:=p_{i} \otimes r_{i}(i=1, \ldots,|\mathcal{G}|)$. Let $S$ be feasible for $(T O P)$ and

$$
R(S)=\frac{1}{|\mathcal{G}|} \sum_{i=1}^{|\mathcal{G}|} P_{i} S P_{i}^{T},
$$

be the Reynolds operator in $S$. Then, $(R(S))$ defines a feasible point for $(T O P)$ with the same objective value as $S$.

Proof Follows directly from Corollary 8.

6.2 Reformulating problem (TOP)

By Corollary 8 we may replace the data matrices in the formulation of (TOP) by their projections onto the centralizer ring to obtain

$$
\begin{array}{ll}
\min & \sum_{i=1}^{m} b_{i} z_{i} \\
\text { s.t. } & S=\sum_{i=1}^{m} R\left(K_{i}-\bar{\Omega} M_{i}\right) z_{i}-\bar{\Omega} R\left(M_{0}\right) \\
& z_{i} \geq 0, \quad i=1, \ldots, m \\
& S \succeq 0,
\end{array}
$$

where the Reynolds operator $R$ is now given by

$$
R(X)=\frac{1}{|\mathcal{G}|} \sum_{i=1}^{|\mathcal{G}|} P_{i} X P_{i}^{T}
$$

where the $P_{i}$ 's are as described in Lemma 9.

Note that each data matrix corresponds to a bar, except for the nonstructural mass matrix $M_{0}$ which is a multiple of the identity.

Now consider a bar $k$. The projection of the data matrix $\left(K_{k}-\bar{\Omega} M_{k}\right)$ onto the commutant depends only on the orbit of bar $k$ under the action of $\mathcal{G}$.

In other words, for two bars $k$ and $k^{\prime}$

$$
R\left(K_{k}-\bar{\Omega} M_{k}\right)=R\left(K_{k^{\prime}}-\bar{\Omega} M_{k^{\prime}}\right)
$$

if and only if $k$ and $k^{\prime}$ share the same orbit. 
We may therefore replace the variables $z_{i}$ that belong to the same orbit $o$ by a single variable $\zeta_{o}$.

$$
\begin{array}{ll}
\min & \sum_{o \text { an orbit }} \zeta_{o} \sum_{i \in o} b_{i} \\
\text { s.t. } & S=\sum_{o \text { an orbit }} \zeta_{o}\left(\sum_{i \in o}\left(K_{i}-\bar{\Omega} M_{i}\right)\right)-\bar{\Omega} R\left(M_{0}\right) \\
& \zeta_{o} \geq 0, \quad o \text { an orbit } \\
& S \succeq 0 .
\end{array}
$$

Note that the matrices $\sum_{i \in o}\left(K_{i}-\bar{\Omega} M_{i}\right)$ lie in the centralizer ring for each orbit $o$.

If we know the irreducible representation of the centralizer ring, we may obtain an orthogonal matrix $Q$ that block-diagonalizes it (see Theorem 3).

Thus we obtain the final formulation

$$
\begin{array}{ll}
\min & \sum_{o \text { an orbit }} \zeta_{o} \sum_{i \in o} b_{i} \\
\text { s.t. } & \sum \zeta_{o \text { an orbit }} Q^{T}\left(\sum_{i \in o}\left(K_{i}-\bar{\Omega} M_{i}\right)\right) Q-\bar{\Omega} Q^{T} R\left(M_{0}\right) Q \succeq 0 \\
& \zeta_{o} \geq 0, \quad o \text { an orbit. }
\end{array}
$$

Note that the number of scalar variables has changed from the number of bars to the number of orbits of bars. The linear matrix inequality has also been block diagonalized, and this may be exploited by interior point solvers for SDP. The actual sizes of the blocks depends on the structure of the group $\mathcal{G}$.

The technique of grouping variables that belong to the same orbits is known as 'design variable linking' in structural optimization, and is often done heuristically. Our description may be seen as a rigorous mathematical basis for this technique.

\section{A $D_{n}$-symmetric dome}

Here we consider a lattice dome truss structure with the dihedral symmetry group $D_{n}$, shown in Fig. 1. (Recall that the dihedral group $D_{n}$ is the symmetry group of an $n$-sided regular polygon for $n>2$.)

The truss in Fig. 1 corresponds to the case $n=6$ (and was studied in Ohsaki 2000), but it is clear that the example may be generalized to all integer values of $n \geq 6$. The free nodes of the truss structure are denoted by filled circles in the figure, and the remaining nodes are fixed. In the general case there will be $4 n$ bars, $n$ fixed nodes and $n+1$ free nodes. Each of the free nodes possesses 3 translational degrees of freedom, giving in total of $3(n+1)$ degrees of freedom for the system.

The symmetry group $D_{n}$ of the truss consists of $n$ elements corresponding to rotations of the polygon, and $n$ more corresponding to reflections. Therefore we may represent the dihedral group in the following way 

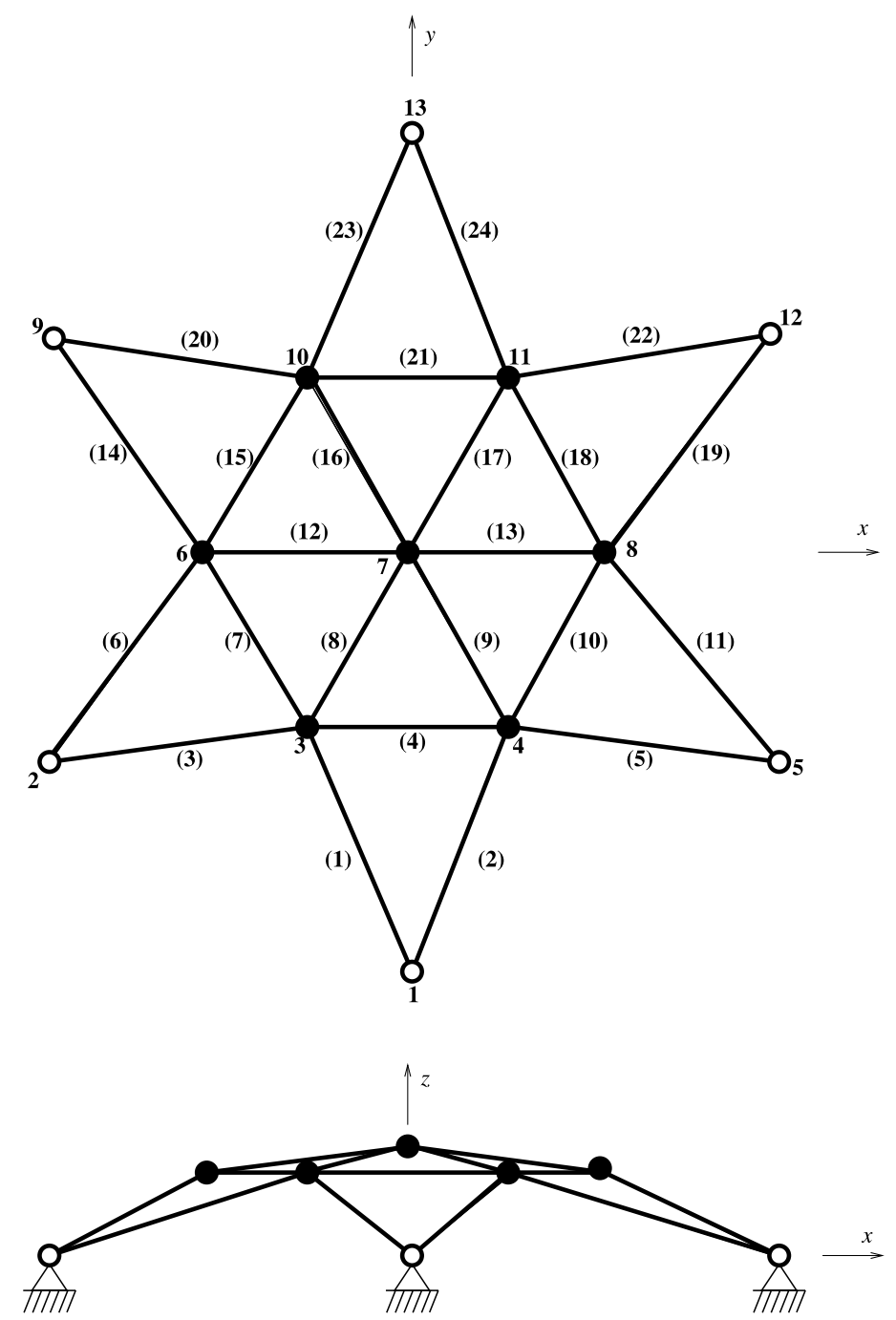

Fig. 1 Top and side views of a spherical lattice dome with $D_{6}$ symmetry. The black nodes are free and the white nodes fixed

$$
D_{n}:=\left\{r\left(\frac{2 \pi}{n} k\right), s r\left(\frac{2 \pi}{n} k\right): k=0,1, \ldots, n-1\right\}
$$

where $r(\alpha)$ stands for counter-clockwise rotation around the $z$-axis at an angle $\alpha$, i.e.

$$
r(\alpha)=\left(\begin{array}{ccc}
\cos (\alpha) & -\sin (\alpha) & 0 \\
\sin (\alpha) & \cos (\alpha) & 0 \\
0 & 0 & 1
\end{array}\right)
$$


Table 1 Comparison of the sizes of the SDP problem before (i.e. (TOP)) and after (i.e. (13)) symmetry reduction, for the dome example (for even $n$ )

\begin{tabular}{lll}
\hline & $\begin{array}{l}\text { \# Scalar } \\
\text { variables }\end{array}$ & p.s.d. matrix variable sizes \\
\hline (TOP) & $4 n$ & $3(n+1) \times 3(n+1)$ \\
$(13)$ & 3 & $1 \times 1,2 \times 2,4 \times 4,3 \times 3(n / 2-1$ times $)$ \\
\hline
\end{tabular}

and $s$ for the reflections with respect to the $x z$-plane, i.e.

$$
s=\left(\begin{array}{ccc}
1 & 0 & 0 \\
0 & -1 & 0 \\
0 & 0 & 1
\end{array}\right)
$$

We proceed to derive the linear orthogonal representation of $\mathcal{G}=D_{n}$ as described in Lemma 9. For continuity of presentation, we only state results here; the details we require on different representations of $D_{n}$ may be found in Appendix.

Our first representation of $D_{n}$ is via the rotation/reflection matrices: for $\alpha=2 \pi / n$, set

$$
r_{i}:=\left\{\begin{array}{l}
r((i-1) \alpha): i=1, \ldots, n \\
\operatorname{sr}((i-(n+1)) \alpha): i=n+1, \ldots, 2 n .
\end{array}\right.
$$

This is the representation $\rho^{1}$ as defined in (15) in Appendix.

Let $p_{i}(i=1, \ldots, 2 n)$ be the permutation matrices that correspond to the permutations $\pi_{i} \in D_{n}$. Thus the representation of $D_{n}$ described in Lemma 9 is given by $P_{i}=p_{i} \otimes r_{i}(i=1, \ldots, 2 n)$.

The size the SDP problem (13), is determined by the number of orbits of bars under the action of $D_{n}$, and the block sizes of the completely reduced representation of the commutant. The number of orbits equals 3 for the example (independent of $n$ ). In particular, the set of bars is tri-partitioned into 3 orbits as follows: those bars connected to a fixed node, those bars connected to the central (hub) node, and the remaining bars (as is clear from Fig. 1).

Moreover, we show in Lemma 12 in Appendix that the block sizes of the commutant are as follows:

1. If $n$ is odd: 1 (one block), 4 (one block) and 3 for the remaining $(n-1) / 2$ blocks;

2. If $n$ is even: 1 (two blocks), 2 (one block), 4 (one block), and 3 for the remaining $n / 2-1$ blocks.

Finally, we compare the sizes of the original problem (TOP) and the reduced problem (13) in Table 1 for even values of $n$.

Note that the biggest gain is in replacing on p.s.d. matrix variable of order $3(n+1)$ by $n / 2+2$ matrix variables of order at most four. This type of block structure can be exploited very well by interior point methods.

Moreover, the number of nonnegative variables was reduced from $4 n$ to only 3 . From a computational viewpoint, problem (13) can easily be solved for $n=1000$, whereas problem (TOP) would be of a size that is very challenging for the present state-of-the-art in interior point method software for semidefinite programming. 


\section{Conclusion and discussion}

We have shown how the semidefinite programming (SDP) formulation of a specific truss topology design problem may be reduced in size by exploiting symmetry conditions. The size reduction has two components: 'variable linking' to eliminate variables and block diagonalization of the SDP data matrices. The former is commonly used in practice in a heuristic way, but the block diagonalization technique is a relatively recent development. The approach we used to reformulate the (SDP) is due to Schrijver (1979, 2005) and described in Gatermann and Parrilo (2004).

An alternative approach for exploiting algebraic symmetry in SDP's was introduced in de Klerk et al. (2007), which uses the so called regular *-representation of the commutant as opposed to its completely reduced representation. The advantage is that the regular $*$-representation can be readily obtained (this is not the case for the irreducible representation). The disadvantage is that it gives less reduction in general than the irreducible representation. Indeed, for the dome example presented in this paper the regular $*$-representation does not give any reduction in problem size.

Acknowledgements The first author kindly acknowledges support of the National Science Foundation of China (No. 10771133) and the program of Shanghai Pujiang (No. 06PJ14039).

Open Access This article is distributed under the terms of the Creative Commons Attribution Noncommercial License which permits any noncommercial use, distribution, and reproduction in any medium, provided the original author(s) and source are credited.

\section{Appendix: Representations and characters of the dihedral group}

A complete description of irreducible $\mathbb{C}$-representations of the dihedral group $D_{n}$ can be found in Serre (1977, Sect. 5.3).

In Murota and Ikeda (1991), $D_{n}$-invariant decompositions of certain $D_{n}$-invariant systems of nonlinear equations arising in bifurcation analysis were studied.

Here we need to adapt the description in Serre (1977, Sect. 5.3) slightly to our case. We are helped by the fact that all the $\mathbb{C}$-representations of $D_{n}$ are in fact equivalent to $\mathbb{R}$-representations. The order of $D_{n}$ is $2 n$, and it is generated by the rotation $r$ through the angle $2 \pi / n$ and any reflection $s$ (i.e. a linear transformation of order 2 fixing a hyperplane (in this case, a line) through the origin) that preserves the regular $n$-gon rotated by $r$. So we know that $r^{n}=s^{2}=(s r)^{2}=1$. Note that it follows that $\left(s r^{k}\right)^{2}=1$ for all $k$. Each element of $D_{n}$ can be uniquely written either in the form $r^{k}$, or in the form $s r^{k}$, for $0 \leq k \leq n-1$.

The irreducible representations of $D_{n} \quad$ The 2-dimensional irreducible $\mathbb{R}$-representation $\rho^{h}$ of $D_{n}$ is given by

$$
\begin{aligned}
& \rho^{h}\left(r^{k}\right)=\left(\begin{array}{cc}
\cos \frac{2 \pi h k}{n} & \sin \frac{2 \pi h k}{n h} \\
-\sin \frac{2 \pi h k}{n} & \cos \frac{2 \pi h k}{n}
\end{array}\right), \quad \rho^{h}(s)=\left(\begin{array}{cc}
1 & 0 \\
0 & -1
\end{array}\right), \\
& \rho^{h}\left(s r^{k}\right)=\rho^{h}(s) \rho^{h}\left(r^{k}\right) .
\end{aligned}
$$


It is straightforward to compute its character

$$
\chi_{h}\left(r^{k}\right)=2 \cos 2 \pi h k / n, \quad \chi_{h}\left(s r^{k}\right)=0 .
$$

It obviously depends upon $h \bmod n$, not $h \in \mathbb{Z}$. Moreover, as $\chi_{h}=\chi_{n-h}$, we have that $\rho^{h}$ is equivalent to $\rho^{n-h}$. Moreover, when $h=0$ or $n=2 h$ the representation $\rho^{h}$ is reducible. So we can take $1 \leq h \leq\lfloor n / 2\rfloor$ to parameterize the representations $\rho^{h}$ uniquely.

The number of 1-dimensional irreducible $\mathbb{R}$-representation $\rho^{h}$ of $D_{n}$ is four, when $n$ is even, and two, when $n$ is odd. In both cases $D_{n}$ has two representations (and the characters, as in the 1-dimensional case it is the same thing) $\psi_{1}$ (the trivial representation), and $\psi_{2}$ given by

$$
\psi_{1}\left(r^{k}\right)=\psi_{1}\left(s r^{k}\right)=1, \quad \psi_{2}\left(r^{k}\right)=-\psi_{2}\left(s r^{k}\right)=1, \quad 0 \leq k \leq n-1 .
$$

In the case $n$ even $D_{n}$ has two more 1-dimensional representations, $\psi_{3}$ and $\psi_{4}$ given by

$$
\psi_{3}\left(r^{k}\right)=\psi_{3}\left(s r^{k}\right)=(-1)^{k}, \quad \psi_{4}\left(r^{k}\right)=-\psi_{4}\left(s r^{k}\right)=(-1)^{k}, \quad 0 \leq k \leq n-1 .
$$

The natural permutation representation $\theta$ of $D_{n} \quad$ We need to determine the decomposition of the character $\chi_{\theta}$ of the permutation representation $\theta$ of $D_{n}$ acting on the $n$-gon into the irreducibles $\psi_{i}$ and $\rho^{h}$ just described. It turns out that they can occur at most once-one says that $\theta$ is multiplicity-free. Indeed, $\theta$ satisfies the well-known sufficient condition for multiplicity-freeness, cf. e.g. Bannai and Ito (1984), that its 2 -orbits, i.e. the orbits on pairs of elements of $\{1, \ldots, n\}$, are symmetric. The latter means that for any $i, j \in\{1, \ldots, n\}$ there exists $g \in D_{n}$ such that $g(i)=j$, and $g(j)=i$. However, not all of $\psi_{i}$ and $\rho^{h}$ will occur-there are simply too many of them. In order to determine the decomposition, we compute $\chi_{\theta}$. After this, we can use Theorem 6 to see which irreducibles occur in $\chi_{\theta}$. (Even without computing, we know that $\psi_{1}$, the trivial character, will occur in $\chi_{\theta}$, as $\theta$ is a permutation representation, so all the group elements fix the sum of coordinates, an invariant 1-dimensional subspace.)

As $\chi_{\theta}$ is the character of a permutation representation, $\chi_{\theta}(g)$ is simply the number of fixed points of $g$, when it is considered as a permutation. So in particular $\chi_{\theta}\left(r^{k}\right)=$ 0 for all $1 \leq k<n$, and $\chi_{\theta}\left(r^{n}\right)=n$. The case $\chi_{\theta}\left(s r^{k}\right)$ needs to be treated separately for $n$ odd, resp. $n$ even. In the former case $\chi_{\theta}\left(s r^{k}\right)=1$.

In the latter case $\chi_{\theta}\left(s r^{k}\right)$ depends upon the parity of $k$. The elements of the form $s r^{k}$ are split into two different conjugacy classes, each of size $n / 2$; the elements of first (resp. second) class fix two opposite vertices (resp. edges) of the $n$-gon, so they have 2 (resp. 0) fixed vertices. So we have $\chi_{\theta}\left(s r^{2 k}\right)=2$ and $\chi_{\theta}\left(s r^{2 k+1}\right)=0$ for $0 \leq k \leq n / 2-1$. To summarize:

$$
\begin{array}{ll}
\chi_{\theta}(1)=n, \quad \chi_{\theta}\left(r^{m}\right)=0, & 1 \leq m \leq n-1, n \in \mathbb{Z}_{+} \\
\chi_{\theta}\left(s r^{k}\right)=1, & 0 \leq k \leq n-1, n \in \mathbb{Z}_{+}-2 \mathbb{Z}, \\
\chi_{\theta}\left(s r^{k}\right)=1+(-1)^{k}, & 0 \leq k \leq n-1, n \in 2 \mathbb{Z}_{+} .
\end{array}
$$


We have $\left\langle\chi_{\theta} \mid \psi_{2}\right\rangle=0$, and Theorem 6 implies that $\psi_{2}$ does not occur in $\theta$. Thus for $n$ odd we obtain

$$
\chi_{\theta}=\psi_{1}+\sum_{h=1}^{(n-1) / 2} \chi_{h}, \quad n \in \mathbb{Z}_{+}-2 \mathbb{Z}
$$

For $n$ even we compute $\left\langle\chi_{\theta} \mid \psi_{3}\right\rangle=1$, and obtain

$$
\chi_{\theta}=\psi_{1}+\psi_{3}+\sum_{h=1}^{n / 2-1} \chi_{h}, \quad n \in 2 \mathbb{Z}_{+} .
$$

9.1 The representation of $D_{n}$ used in the dome truss example

Here, we construct the representation $\mathcal{P}$ of $\mathcal{G}=D_{n}$ as

$$
\mathcal{P}=\left(\psi_{1} \oplus \theta\right) \otimes\left(\psi_{1} \oplus \rho^{1}\right),
$$

where, as before, $\psi_{1}$ denotes the trivial 1-dimensional representation, $\theta$ the natural permutation representation, and $\rho^{1}$ is as in (15). Note that the representation $\psi_{1} \oplus \rho^{1}$ is equivalent to the one described in (14) for $\alpha=2 \pi / n$.

In order to analyze the block structure of the commutant, as described in Theorem 14 , we should find a decomposition of $\mathcal{P}$ into irreducibles. It suffices to compute the decomposition of the character $\chi_{\mathcal{P}}$ into irreducible characters using (3). Thus, expanding the tensor product, we obtain

$$
\mathcal{P}=\psi_{1} \oplus \theta \oplus \rho^{1} \oplus \bigoplus_{h}\left(\rho^{h} \otimes \rho^{1}\right) \oplus \rho^{1} \oplus\left(\psi_{3} \otimes \rho^{1}\right) .
$$

The $\oplus$-summation index $h$ ranges as in (16) for $n$ odd, resp. as in (17) for $n$ even, using $t:=n$. The last term in this decomposition is 0 when $t$ is odd. Otherwise, computing the character,

$$
\left(\chi_{1} \otimes \psi_{3}\right)\left(r^{k}\right)=2(-1)^{k} \cos \frac{2 \pi k}{n}=2 \cos \left(\pi k-\frac{2 \pi k}{n}\right)=\chi_{\frac{n}{2}-1}\left(r^{k}\right),
$$

we obtain $\psi_{3} \otimes \rho^{1}=\rho^{\frac{n}{2}-1}$. Similarly one computes

$$
\begin{aligned}
\left(\chi_{1} \otimes \chi_{h}\right)\left(r^{k}\right) & =4 \cos \frac{2 \pi k}{n} \cos \frac{2 \pi h k}{n}=2 \cos \frac{2 \pi(h+1) k}{n}+2 \cos \frac{2 \pi(h-1) k}{n} \\
& =\left(\chi_{h+1}+\chi_{h-1}\right)\left(r^{k}\right),
\end{aligned}
$$

deriving $\rho^{h} \otimes \rho^{1}=\rho^{h+1} \oplus \rho^{h-1}$ as long as $\chi_{h+1}$ are $\chi_{h-1}$ are defined and irreducible. When $h=1$ we further decompose $\rho^{h-1}=\psi_{1} \oplus \psi_{2}$. When $n$ is even and $h=n / 2-1$ we further decompose $\rho^{n / 2}=\psi_{3} \oplus \psi_{4}$, and when $n$ is odd and $h=(n-1) / 2$ we get $\rho^{h+1}=\rho^{n-h-1}=\rho^{(n-1) / 2}$.

To summarize, we have the following proposition. 
Lemma 12 Consider the representation $\mathcal{P}$ of $D_{n}$, given by (18).

For $n$ odd, one has:

$$
\mathcal{P}=3 \psi_{1} \oplus \psi_{2} \oplus \rho^{1} \oplus 3 \bigoplus_{h=1}^{(n-1) / 2} \rho^{h}
$$

The block sizes of the commutant are thus 1 (for $\left.\psi_{2}\right), 4\left(\right.$ for $\left.\rho^{1}\right)$ and 3 for the remaining $(n-1) / 2$ irreducibles. Respectively, the dimension of the commutant is $1+4^{2}+3^{2}(n-1) / 2$.

For $n$ even, one has:

$$
\mathcal{P}=3 \psi_{1} \oplus \psi_{2} \oplus 2 \psi_{3}+\psi_{4} \oplus \rho^{1} \oplus 3 \bigoplus_{h=1}^{n / 2-1} \rho^{h} .
$$

The block sizes of the commutant are thus 1 (for $\psi_{2}$ and $\psi_{4}$ ), 2 (for $\psi_{3}$ ), 4 (for $\rho^{1}$ ), and 3 for the remaining $n / 2-1$ irreducibles. Respectively, the dimension of the commutant is $2+2^{2}+4^{2}+3^{2}(n / 2-1)$.

\section{References}

Bannai E, Ito T (1984) Algebraic combinatorics I: association schemes. Benjamin/Cummings, Redwood de Klerk E, Maharry J, Pasechnik DV, Richter B, Salazar G (2006) Improved bounds for the crossing numbers of $K_{m, n}$ and $K_{n}$. SIAM J Discr Math 20:189-202

de Klerk E, Pasechnik DV, Schrijver A (2007) Reduction of symmetric semidefinite programs using the regular *-representation. Math Program B 109(2-3):613-624

Gatermann K, Parrilo PA (2004) Symmetry groups, semidefinite programs, and sum of squares. J Pure Applies Algebra 192:95-128

Graham A (1981) Kronecker products and matrix calculus with applications. Ellis Horwood, Chichester

Kanno Y, Ohsaki M, Murota K, Katoh N (2001) Group symmetry in interior-point methods for semidefinite program. Optim Eng 2(3):293-320

Miller W (1972) Symmetry, groups and their applications. Academic Press, San Diego

Murota K, Ikeda K (1991) Computational use of group theory in bifurcation analysis of symmetric structures. SIAM J Sci Stat Comput 12:273-297

Ohsaki M (2000) Optimization of geometrically non-linear symmetric systems with coincident critical points. Int J Numer Methods Eng 48:1345-1357

Ohsaki M, Fujisawa K, Katoh N, Kanno Y (1999) Semi-definite programming for topology optimization of trusses under multiple eigenvalue constraints. Comp Methods Appl Mech Eng 180:203-217

Schrijver A (1979) A comparison of the Delsarte and Lovász bounds. IEEE Trans Inf Theory 25:425-429

Schrijver A (2005) New code upper bounds from the Terwilliger algebra. IEEE Trans Inf Theory 51:28592866

Serre J-P (1977) Linear representations of finite groups. Graduate texts in mathematics, vol 42. Springer, New York

Wedderburn JHM (1907) On hypercomplex numbers. Proc Lond Math Soc 6(2):77-118

Wedderburn JHM (1934) Lectures on matrices. AMS colloquium publications, vol 17, AMS publishers 